\title{
ELLIPTICAL CAVITY SHAPE OPTIMIZATION FOR ACCELERATION AND HOM DAMPING*
}

\author{
Haipeng Wang ${ }^{\#}$, Robert Rimmer, Genfa Wu, TJNAF, Newport News, VA, 23606
}

\section{Abstract}

We report a survey of center cell shapes developed for Superconducting Radio Frequency (SRF) multi-cell cavities for different projects. Using a set of normalized parameters, we compare the designs for different frequencies and particle velocities for the fundamental mode. Using dispersion curves of High Order Modes (HOM) (frequency verse phase advance) calculated by MAFIA for a single cell, we further optimize the cavity shape to avoid a light cone line crossing at the dangerous resonance frequencies determined by the beam bunch structure and eliminate the trapped (or high $\mathrm{R} / \mathrm{Q}$ ) modes with a low group velocity. We developed this formulation to optimize a 5 -cell, $750 \mathrm{MHz}$ cavity shape, with good real-estate accelerating gradient and a strong HOM damping waveguide structure for the JLab 1MW ERLFEL project.

\section{INTRODUCTION}

A traditional design process for a SRF cavity shape is to maximize the $\mathrm{R} / \mathrm{Q}$ of fundamental mode (shunt impedance/intrinsic quality factor) and geometry factor $\mathrm{G}$ for a given RF field limit of $\mathrm{B}_{\text {peak }} / \mathrm{E}_{\text {acc }}$ or $\mathrm{E}_{\text {peak }} / \mathrm{E}_{\text {acc. }}$. For a high average beam current Energy Recovery Linac (ERL) like the Free Electron Laser (FEL) at JLab, any HOM frequency coincident with a resonance of the beam or optics structure could cause a huge power deposition or beam breakup (BBU). It is critical to consider HOM damping and resonance avoidance in the design optimization. With a given cavity shape and HOM coupling, beam-induced voltages can be calculated and the narrow band cavity impedance [1] and BBU threshold [2] can be estimated. These processes are usually applied only after cavity manufacture, and the results can only benefit future designs. Dispersion curve modeling has been used for traveling-wave, normal-conducting, multicell cavities for a long time. Recently it has been used for the HOM studies of a superconducting cavity [3]. We found that this method can be extended to calculate the $\mathrm{R} / \mathrm{Q}$ and group velocity of each passband of HOM and the resonant frequency of a trapped HOM can be predicted. A MAFIA [4] 2D eigen-mode solver with periodic boundary conditions has been extensively used in this analysis. The SRF parameters of the fundamental mode were confirmed by SUPERFISH [5]. Finally, single bunch loss factors were calculated by the ABCI94 code [6].

\section{CAVITY SHAPE DESIGN SURVEY}

\footnotetext{
* Supported by the Office of Naval Research, the Joint Technology Office, the Commonwealth of Virginia, the Air Force Research Laboratory, and by DOE Contract DE-AC05-84ER40150.

\# E-mail: haipeng@jlab.org
}

Various designs of SRF cavities have been used for different acceleration frequencies and particle velocities. We surveyed only inner cell shapes using a normalized coordinate in Figure 1 and a normalized parameter set in Table 1. They are cell shape dependent only, so a fair comparison is possible. The name ID represents different projects referenced in Table 1 . The first ten rows are from developed projects [7-17]. The next three rows are the projects currently under development. The next four shapes starting from Rounded Pillbox (RP) are the designs under this optimization. The data from the Pillbox and Hemi-sphere are analytical results representing theoretical limits with zero iris and no cell-to-cell coupling $\left(\mathrm{k}_{\mathrm{cc}}=0\right)$. Figure 1 only draws $\beta=1$ shapes for clarity, but $\beta<1$ shapes can be plotted in same graph.

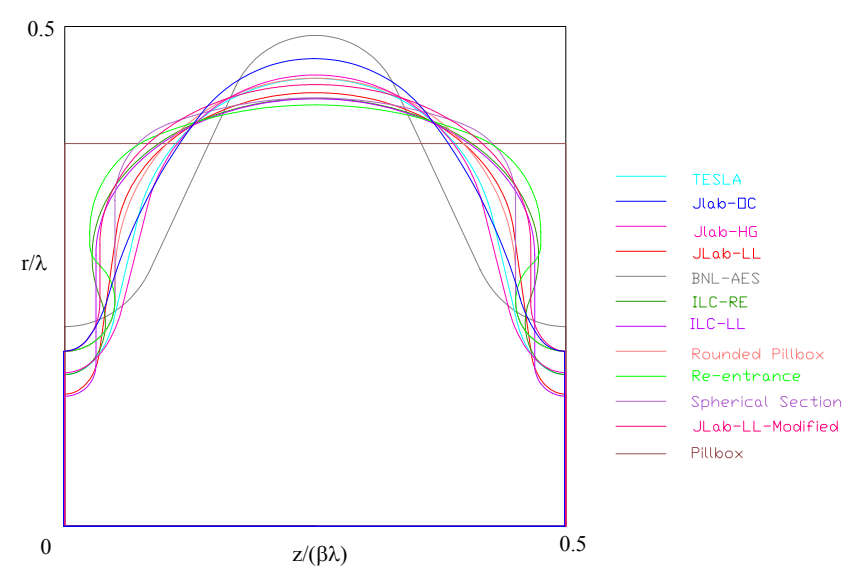

Figure 1: Cavity center cell shapes from different projects. Here $\lambda$ is fundamental mode wavelength, $\beta=\mathrm{v} / \mathrm{c}$ is designed particle relative velocity.

Our preference for high-current applications, from Table 1, is a low loss (LL) design for low cryogenic cost in CW operation. Either JLab-LL or ILC-LL shape has relatively high $\mathrm{R} / \mathrm{Q}$ and $\mathrm{G}$. Other designs optimized for low- $\beta$ beam or pulsed-mode operation, were excluded. Re-entrant shapes are not yet practical for chemical cleaning. To preserve the high $\mathrm{R} / \mathrm{Q}$ in the LL shapes, a small iris ratio $r / \lambda$ is needed.

\section{HOM POWER DEPOSITION}

Due to the high beam power, any high impedance HOM that resonates with the bunch excitation will cause a huge power deposition.

\section{Power Spectrum}

A time averaged HOM power spectrum has been calculated for the pulsed proton beam structure in the Spallation Neutron Source (SNS) linac [18]. This algorithm has been modified for the ERL-FEL beam 
Table 1: Normalized fundamental mode RF parameter comparison for various cavity shapes, some of which are shown in Figure 1. $\mathrm{R} / \mathrm{Q}=\mathrm{V}_{\mathrm{a}}{ }^{2} / \omega_{0} \mathrm{U}$, where $\mathrm{V}_{\mathrm{a}}$ is the beam voltage including transit time factor. $\omega_{0}=2 \pi \mathrm{f}_{0}$. $\mathrm{U}$ is total stored energy.

\begin{tabular}{|c|c|c|c|c|c|c|c|c|c|c|}
\hline Name ID & $\begin{array}{c}\text { Freq.. } \\
{[\mathbf{M H z}]}\end{array}$ & $\begin{array}{c}\boldsymbol{\beta} \\
=\mathbf{v} / \mathbf{c}\end{array}$ & $\begin{array}{c}\mathbf{C e l l} \\
\mathbf{N o} \\
\mathbf{N}_{\mathbf{c}}\end{array}$ & $\begin{array}{c}\mathbf{R} /\left(\mathbf{Q} \boldsymbol{\beta}^{\mathbf{2}} \mathbf{)}\right. \\
\mathbf{/ \mathbf { c e l l }} \\
{[\mathbf{\Omega} / \mathbf{c e l l}]}\end{array}$ & $\begin{array}{c}\mathbf{E}_{\text {peak }} \\
/ \mathbf{E}_{\text {acc }}\end{array}$ & $\begin{array}{c}\mathbf{B}_{\text {peak }} / \mathbf{E}_{\text {acc }} \\
[\mathbf{m T} \mathbf{T} / \mathbf{M V} / \mathbf{m})]\end{array}$ & $\begin{array}{c}\mathbf{G} \\
{[\Omega]}\end{array}$ & $\begin{array}{c}\text { Equator } \\
\mathbf{R} / \boldsymbol{\lambda}\end{array}$ & $\begin{array}{c}\mathbf{I r i s} \\
\mathbf{r} / \boldsymbol{\lambda}\end{array}$ & $\begin{array}{c}\mathbf{k}_{\mathbf{c c}} \\
{[\mathbf{\%}]}\end{array}$ \\
\hline APT-med $\beta[7]$ & 700 & 0.64 & 5 & 82.3 & 2.94 & 7.10 & 162 & 0.466 & 0.152 & 3.02 \\
\hline APT-hi $\beta[7]$ & 700 & 0.82 & 5 & 77.6 & 2.48 & 6.10 & 213 & 0.469 & 0.187 & 4.41 \\
\hline SNS-med $\beta[8]$ & 805 & 0.61 & 6 & 125.0 & 2.71 & 5.72 & 179 & 0.440 & 0.115 & 1.53 \\
\hline SNS-hi $\beta[8]$ & 805 & 0.81 & 6 & 122.7 & 2.19 & 4.72 & 260 & 0.440 & 0.131 & 1.52 \\
\hline RIA [9] & 805 & 0.47 & 6 & 120.7 & 3.41 & 6.90 & 136.7 & 0.464 & 0.104 & 1.50 \\
\hline TRASCO [10] & 700 & 0.47 & 5 & 167.8 & 2.90 & 5.38 & 147.8 & 0.440 & 0.093 & 1.35 \\
\hline TESLA [11] & 1300 & 1 & 9 & 115.1 & 2.0 & 4.26 & 270 & 0.448 & 0.152 & 1.87 \\
\hline JLab-OC [12] & 1497 & 1 & 7 & 96.0 & 2.56 & 4.56 & 273.8 & 0.468 & 0.175 & 3.29 \\
\hline JLab-HG [13] & 1497 & 1 & 7 & 111.9 & 1.89 & 4.26 & 265.5 & 0.451 & 0.153 & 1.72 \\
\hline JLab-LL [14] & 1497 & 1 & 7 & 128.8 & 2.17 & 3.74 & 280.3 & 0.434 & 0.132 & 1.49 \\
\hline BNL-AES [15] & 704 & 1 & 5 & 79.4 & 1.99 & 5.82 & 225 & 0.491 & 0.200 & 3.16 \\
\hline ILC-RE [16] & 1300 & 1 & 8 & 126.8 & 2.19 & 3.78 & 278 & 0.428 & 0.152 & 2.38 \\
\hline ILC-LL [17] & 1300 & 1 & 9 & 133.7 & 2.51 & 3.58 & 284 & 0.428 & 0.130 & 1.54 \\
\hline Rounded Pillbox & 750 & 1 & 5 & 103.5 & 2.40 & 4.26 & 279.7 & 0.449 & 0.175 & 3.18 \\
\hline Re-entrance & 750 & 1 & 5 & 107.6 & 2.66 & 4.52 & 202.9 & 0.422 & 0.175 & 3.52 \\
\hline Spherical Section & 750 & 1 & 5 & 105.3 & 2.47 & 4.32 & 273.0 & 0.429 & 0.175 & 3.31 \\
\hline JLab-LL-mod. & 750 & 1 & 5 & 101.3 & 2.91 & 4.14 & 291.1 & 0.442 & 0.175 & 4.20 \\
\hline Pillbox & - & 1 & 1 & 195.9 & 1.57 & 3.05 & 256.6 & 0.383 & 0 & 0 \\
\hline Hemi-sphere & - & 0.873 & 1 & 191.2 & 1.90 & 3.69 & 216.0 & 0.437 & 0 & 0 \\
\hline
\end{tabular}

structure to account for both accelerating and decelerating bunches [19]. A point charge repeatedly charges an RF cavity with a constant damping but a variable recirculated sub-time structure. Such a power spectrum depends on the beam (bunch charge, length, photocathode laser frequency in $\mathrm{CW}$ or pulsed mode and recirculation time) and cavity (HOM frequency, R/Q and external Q). Comparing with one-beam-pass machine, an ERL's power spectrum will have anti-resonance nodes on odd harmonics of cavity fundamental frequency. But sidebands at harmonics of the laser frequency will resonate quadruply in amplitude due to the two-beam scheme. Figure 2 shows a semi-log plot of this spectrum normalized to $\mathrm{R} / \mathrm{Q}$ (in blue). With a $1.33 \mathrm{nC} /$ bunch charge (1A at $750 \mathrm{MHz}$ ), higher average current gives the higher peaks. The $\mathrm{Q}_{\mathrm{ext}}$ is proportional to the peaks. A low $\mathrm{Q}_{\mathrm{ext}}$ will raise tails portion of the spectrum. When the $Q_{e x t}$ is low enough $(\sim 10)$, a broad band spectrum is expected as a single bunch loss effect.

\section{Dispersion Curves}

The continuous dispersion curves (phase advance verse frequency, $X-Y$ rotated) between 0 to $\pi$ represent the $\mathrm{HOM}$ passbands in an infinite number $\mathrm{N}_{\mathrm{c}}$ of a periodic cell structure. With a finite $\mathrm{N}_{c}$, the passband frequencies will be discrete, but this continuum approximation is useful. The crossing points between the dispersion curves and the folded light cone lines (thick red in Figures 2 and 3 ), called synchronous points, are where the RF phase velocity is as same as the speed of light or the electron beam speed. Maximum transit time factor (or R/Q) within the passband occurs at these points. In Figure 2, we overlap the dispersion curves of TM monopole modes on the power spectrum and examine any crossing point both near resonance peak lines (vertical) and the grid lines representing the five passband modes of our 5-cell cavity. Those points are high $\mathrm{R} / \mathrm{Q}$ modes. These points are as many as upper limits of above the cut-off frequencies. Within those modes, most dangerous ones are those " 0 " (phase advance) modes. They near both the resonance lines and regions of zero group velocity $(\mathrm{df} / \mathrm{d} \Phi=0)$. Zero group velocity is standing wave or trapped mode. They are not easily damped in a long ( $>5$-cell) structure.

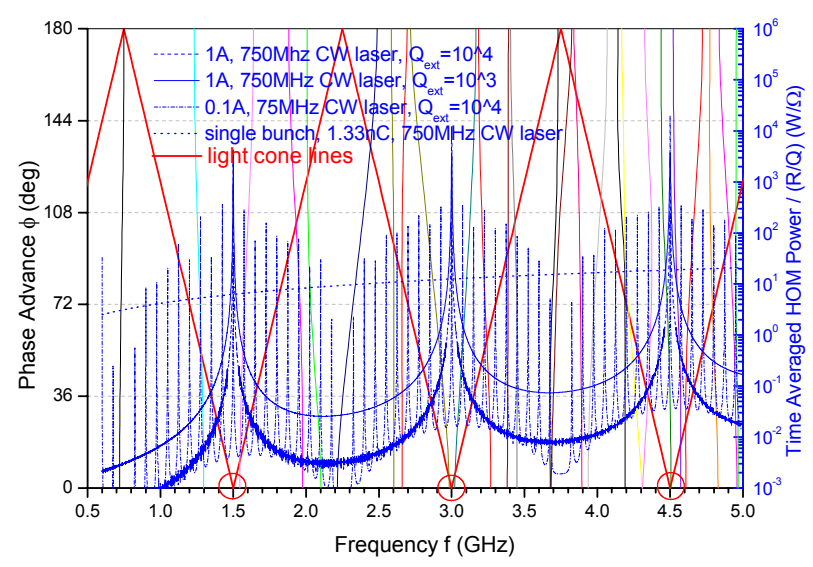

Figure 2: TM monopole HOM resonance avoidance design for a modified JLab Low Loss cavity shape.

In Figure 3, we zoom in on the plot near $3 \mathrm{GHz}$ and compare different cavity shapes. For HOM damping to $\mathrm{Q}_{\mathrm{ext}}=1 \mathrm{e} 3,750 \mathrm{~W}$ will be deposited in a 5-cell structure of the RP shape. If due to the cavity manufacturing and tuning errors, this TM030 mode were to overlap the 3 
$\mathrm{GHz}$ beam harmonic, up to $19 \mathrm{~kW}$ of HOM power could be deposited which would be a challenge for the damper. Careful control of this frequency is essential for machine protection. A correct way to calculate the $\mathrm{R} / \mathrm{Q}$ is using the phase value only at the synchronous point as the input for the periodic phase advance in MAFIA. The RF fields calculated are complex, representing energy exchange through the phase boundaries. The $\mathrm{R} / \mathrm{Q}$ has to involve the modulus of integration over these complex fields [18].

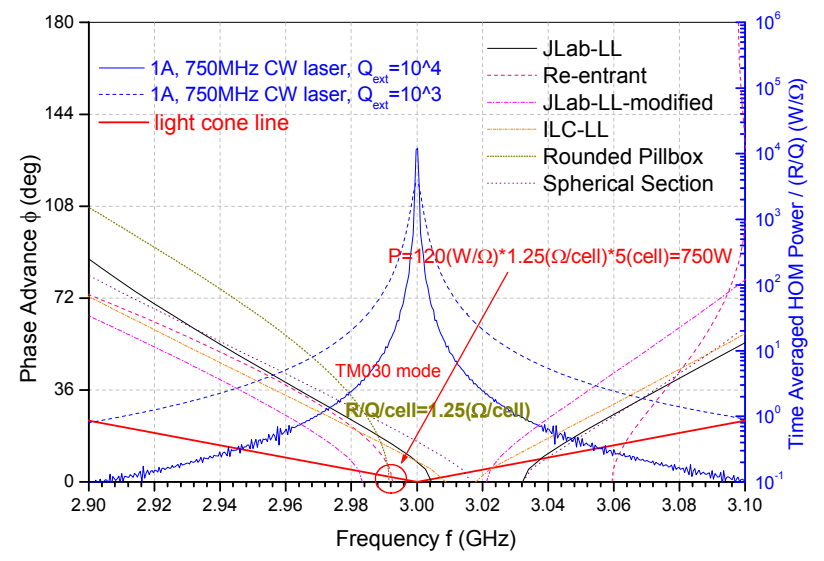

Figure 3: TM030 trapped mode in different cavity shapes and its power calculation for a 5-cell RP shape cavity.

Because of the exponential scale of the power spectrum, in the second order optimization, we chose the cavity shape having the "0" mode points away from the $1.5,3.0$ and $4.5 \mathrm{GHz}$ harmonic lines to avoid large power deposition. Table 2 shows the options. We found that the " 0 " mode for the RP shape has a relative lower R/Q than other shapes at $3 \mathrm{GHz}$. A 3D MAFIA simulation of a 5cell RP shape, with waveguides damping structures confirmed that the impedance at $3 \mathrm{GHz}$ is reduced relative to other shapes. At $4.5 \mathrm{GHz}$, the JLab-LL-modified shape has a closed $\mathrm{df} / \mathrm{d} \phi$ value (not shown) to the RP shape at $3 \mathrm{GHz}$. Similar calculations were done for all dipole modes, but the power deposited will be normally lower than the monopole modes by a factor of 10 to 100 and we do not consider them further in the design.

\section{BBU AND SINGLE BUNCH LOSS}

The BBU current threshold depends on the dipole modes parameters and the beam optics [20]. With a 50 meter of beam re-circulation path, the unstable beam grid lines will be only $3 \mathrm{MHz}$ apart. Mechanical errors could easily cause HOM frequency change that large, so it is not practical to optimize the cavity shape to accommodate this constraint.

Both JLab-LL and ILC-LL low loss shapes have a smaller iris. This leads to trapped HOMs and a high single bunch loss factor $\mathrm{k}$. We increase the iris to $14 \mathrm{~cm}$ with a compromise in $\mathrm{R} / \mathrm{Q}$ but preserve a high $\mathrm{G}$ factor. In Table 2 , we include integrated $\mathrm{k}$ up to $120 \mathrm{GHz}$, where $\mathrm{k}_{\|}$is for the longitudinal loss and $k_{\perp}$ is for the transverse kick.
They agree with the scaling law [21] of $k_{\|} \propto \frac{1}{r} \sqrt{\frac{R N_{c}}{\sigma_{z}}}$, $k_{\perp} \propto \frac{1}{r^{3}} \sqrt{R N_{c} \sigma_{z}} \cdot \sigma_{\mathrm{z}}$ is beam rms bunch length. Final four shapes in Table 2 would give $7.5 \mathrm{~kW}$ broad-band power loss per cavity for a $1.33 \mathrm{nC} /$ bunch, $750 \mathrm{MHz} \mathrm{CW}$ beam. This power can travel either direction to the beam pipe. We have to design a warm HOM load at each end of the cryomodule to absorb this HOM power.

Table 2: HOM calculation for selected cavity shapes scaled to $750 \mathrm{MHz}$. The $\mathrm{k}$ loss factors are for $\sigma_{\mathrm{z}}=1 \mathrm{~mm}$.

$\begin{array}{cccccc}\text { Name } & \mathrm{k}_{/ /} / \text {cell } & \mathrm{k}_{\perp} / \text { cell } & \Delta \mathrm{f} \text { to } & \Delta \mathrm{f} \text { to } & \Delta \mathrm{f} \text { to } \\ \text { ID } & {[\mathrm{V} / \mathrm{pC}} & {[\mathrm{V} / \mathrm{pC}} & 1.5 \mathrm{Ghz} & 3 \mathrm{Ghz} & 4.5 \mathrm{Ghz} \\ & / \mathrm{cell}] & / \mathrm{m} / \mathrm{cell}] & {[\mathrm{MHz}]} & {[\mathrm{MH}]} & {[\mathrm{MHz}]} \\ & 1.15 & 0.65 & -31.9 & -62.4 & -170.9 \\ \text { ab-OC } & 1.49 & 1.41 & 89.8 & 4.43 & 4.73 \\ \text { ab-LL } & 1.46 & 0.22 & - & - & - \\ \text { C-LL } & 1.48 & 1.41 & 1145 & 8.80 & -9.30 \\ \text { und. Pill. } & 1.12 & 0.59 & 29.7 & -8.47 & -157.4 \\ \text {-entrant } & 1.12 & 0.59 & 1242 & -7.45 & 23.4 \\ \text { phere. Sect. } & 1.12 & 0.59 & 86.2 & 16.5 & 24.2 \\ \text { ab-LL-mod. } & 1.14 & 0.60 & 46.8 & 22.4 & 5.49\end{array}$

\section{CONCLUSION}

Both Rounded Pillbox and JLab-LL-modified shapes are candidates for a 5-cell cavity for the JLab 1MW ERLFEL project. Mutipactor simulations (MultiPac 2.1) on single cells of both cavities show no multiplication of secondary electrons with impact energy above $35 \mathrm{eV}$.

\section{REFERENCES}

[1] R. A. Rimmer et al, Proc. PAC2003, p1389-1391.

[2] K. Beard et al, Proc. PAC2003, p332-334.

[3] T. Khabibouline et al, Fermi Lab Tech Note TM2210, May, 2003.

[4] MAFIA, CST, http://www.cst.com.

[5] SUPERFISH, LLACG, http://laacg.lanl.gov/laacg/.

[6] Y Chin, CERN Tech Note SL/94-02 (AP).

[7] F. Krawczyk et al, Proc. PAC1997, p2914-2916.

[8] S. Kim et al, Proc. LINAC2000, p923-925.

[9] G. Ciovati et al, JLab Tech Note 01-014.

[10]D. Barni et al, Proc. EPAC2002, p2277-2279.

[11] B. Aune et al, PRST-AB, Vol. 3092001 (2000).

[12]R. Sundelin, Cornell Univ. Tech Note SRF-820803.

[13]C. Ciovati et al, JLab Tech Note 01-015.

[14] J. Sekutowicz et al, JLab Tech Note 02-023.

[15]R. Calaga, presented at SRF2003 Workshop, Sep. 812, 2003, Lubeck Germany.

[16]J. Sekutowicz, presented at ILC Workshop, KEK Japan, Nov. 13-15, 2004.

[17] H. Shemelin et al., NIM-PR A 496 (2003), p1-7.

[18] S. Kim et al, NIM-PR A 492 (2002), p1-10.

[19] H. Wang, JLab Tech Note 05-047.

[20]E. Pozdeyev et al, JLab Tech Note 04-019.

[21]R Calaga et al, presented at ERL2005 Workshop, JLab, March 20, 2005. 